\title{
Fracture evaluation of metallic materials at intermediate strain rates
}

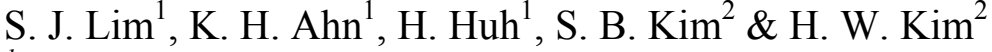 \\ ${ }^{I}$ School of Mechanical, Aerospace and System Engineering, \\ Republic of Korea \\ ${ }^{2}$ Agency for Defense Development, Republic of Korea
}

\begin{abstract}
This paper evaluates existing dynamic hardening models and fracture strains for metallic materials of typical crystalline structures: 4130 steel (BCC); OFHC copper (FCC); and Ti6Al4V (HCP). Uniaxial tensile and fracture tests of the representative materials are carried out at intermediate strain rate. In order to describe the dynamic hardening characteristics, several models are compared for each material and the most applicable model for a specific material has been recommended. The fracture tests at strain rates ranging from $0.001 \mathrm{~s}^{-1}$ to $100 \mathrm{~s}^{-1}$ are conducted with several types of the pure shear, dog bone and grooved specimens for the strain paths of the pure shear, uniaxial tension and plain strain conditions. The fracture strain and the strain path are measured on the surface of the specimen and the Lou-Huh ductile fracture criterion is implemented to describe the fracture loci with respect to the strain rate.

Keywords: ductile fracture criterion, strain rate, 4130 steel, OFHC copper, Titanium alloy.
\end{abstract}

\section{Introduction}

Military weapons such as a missile explode under high impact and pressure conditions. Because weapons are designed using numerical simulations in order to save time and cost, the accurate material properties need to be secured under the extreme condition. In this paper, the uniaxial tensile and fracture tests of 4130 steel (BCC), OFHC copper (FCC) and Ti6Al4V (HCP) at strain rates ranging from $0.001 \mathrm{~s}^{-1}$ to $100 \mathrm{~s}^{-1}$ are executed as the first work of measuring the dynamic material properties of representative materials for a warhead. 
Many researchers tried to describe the dynamic hardening characteristics of metallic materials with hardening models such as the Johnson-Cook model, Zerilli-Armstrong model and Preston-Tonks-Wallace [1] model. However, no model is accurately applicable for all materials, because each model approximates the hardening curve with the different characteristics as shown in table 1. Therefore, the applicable models have to be recommended for 4130 steel, OFHC copper and Ti6Al4V in order to be implemented in numerical simulation.

Table 1: $\quad$ The characteristics of typical dynamic hardening models.

\begin{tabular}{|c|c|c|c|}
\hline & \multicolumn{2}{|c|}{$\begin{array}{l}\text { Approximation characteristics } \\
\text { w.r.t the strain rate }\end{array}$} \\
\hline & & Yield stress & Flow stress \\
\hline \multicolumn{2}{|c|}{ Johnson-Cook model } & Linear function & Proportional \\
\hline \multirow{2}{*}{$\begin{array}{c}\text { Zerilli-Armstrong } \\
\text { model }\end{array}$} & BCC model & Exponential function & Independent \\
\hline & FCC model & Independent constant & Proportional \\
\hline \multicolumn{2}{|c|}{ Preston-Tonks-Wallace model } & Error function & Flexible \\
\hline \multicolumn{2}{|c|}{ Modified Johnson-Cook model } & Exponential function & Proportional \\
\hline \multicolumn{2}{|c|}{ Lim-Huh model } & Exponential function & Flexible \\
\hline \multicolumn{2}{|c|}{ Modified Khan-Huang model } & Exponential function & Flexible \\
\hline
\end{tabular}

Fracture characteristics is one of the important material properties to simulate the warhead penetration. The warhead undergoes a wide range of stress state from compressive upsetting to the balanced biaxial tension during the penetration. Because the fracture strain changes with respect to the loading path and the strain rate, fracture tests also should be conducted with respect to the loading path and strain condition. Many ductile fracture criteria have been proposed in the last decade, but those are not accurately applicable for all materials. In order to expand the generality of a ductile fracture criterion, the Mohr-Coulomb criterion and the Lou-Huh ductile fracture criterion are suggested in the space of stress triaxiality, the Lode parameter and equivalent fracture strain adopting the calibration method. The critical difference between two criteria is that a cut-off value for the stress triaxiality is extremely small for the Mohr-Coulomb criterion [2, 3] while the Lou-Huh criterion [4] proposes a constant cut-off value of $-1 / 3$ which is reasonable for the ductile material such as warhead.

\section{Experimental conditions}

\subsection{Materials}

For the tests, 4130 steel (Calstrip Steel Corporation), OFHC copper (Aurubis) and Ti6Al4V (TIMET) sheets with $1.2 \mathrm{~mm}$ thickness are used to measure the dynamic hardening properties and the fracture strains. The sheets were prepared according to AMS 6350 and the chemical composition of each material is shown in table 2 . 
Table 2: $\quad$ Chemical composition of test materials.

4130 steel

\begin{tabular}{|c|c|c|c|c|c|}
\hline Element & Mn & C & Cr & Mo & Si \\
\hline $\mathrm{wt} \%$ & 0.5 & 0.32 & 0.95 & 0.19 & 0.19 \\
\hline
\end{tabular}

OFHC copper

\begin{tabular}{|c|c|c|c|c|c|}
\hline Element & $\mathbf{A g}$ & $\mathbf{S}$ & $\mathbf{N i}$ & $\mathbf{F e}$ & $\mathbf{A s}$ \\
\hline $\mathrm{wt} \%$ & 0.0025 & 0.0015 & 0.001 & 0.001 & 0.0006 \\
\hline
\end{tabular}

Ti6Al4V

\begin{tabular}{|c|c|c|c|c|c|}
\hline Element & Al & V & Fe & O & C \\
\hline $\mathrm{wt} \%$ & 6.21 & 4.02 & 0.17 & 0.11 & 0.009 \\
\hline
\end{tabular}

\subsection{Specimen shapes and tensile speeds}

Many researchers have proved that the fracture strain depends on the stress triaxiality and the strain path. As excluding the hysteresis effect, the shape of a tensile specimen has to induce a proportional loading path. In order to realize three typical strain paths, the pure shear, dog bone and grooved specimens are used for the strain paths of the pure shear, uniaxial tension and plain strain conditions as shown in Figs 1 and 2. The result with the notched specimen is utilized not to calibrate the Lou-Huh model but to evaluate the validity of the model. In order to measure the fracture strain on the specimen surface, black spackle patterns are sprayed on the white background. Whole deformation during the test is recorded by a high speed camera and the fracture strain before fracture is calculated using a digital image correlation (DIC) method with a commercial

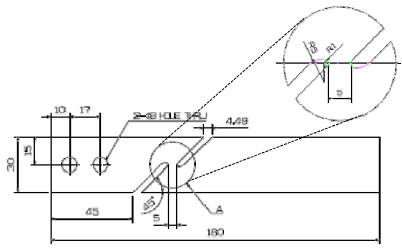

(a)

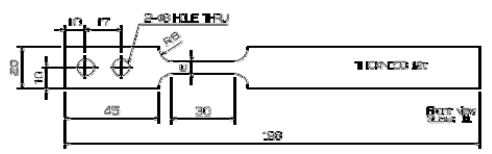

(c)

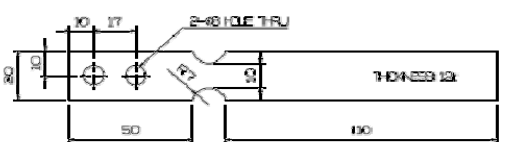

(b)

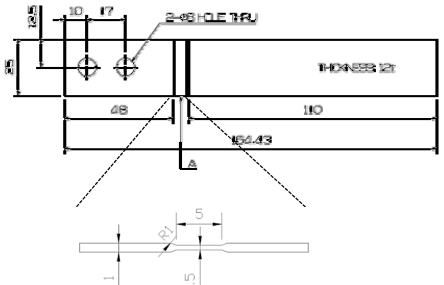

(d)

Figure 1: $\quad$ Specimen dimensions for the fracture test; (a) pure shear specimen; (b) notched specimen; (c) dog bone specimen; (d) grooved specimen. 


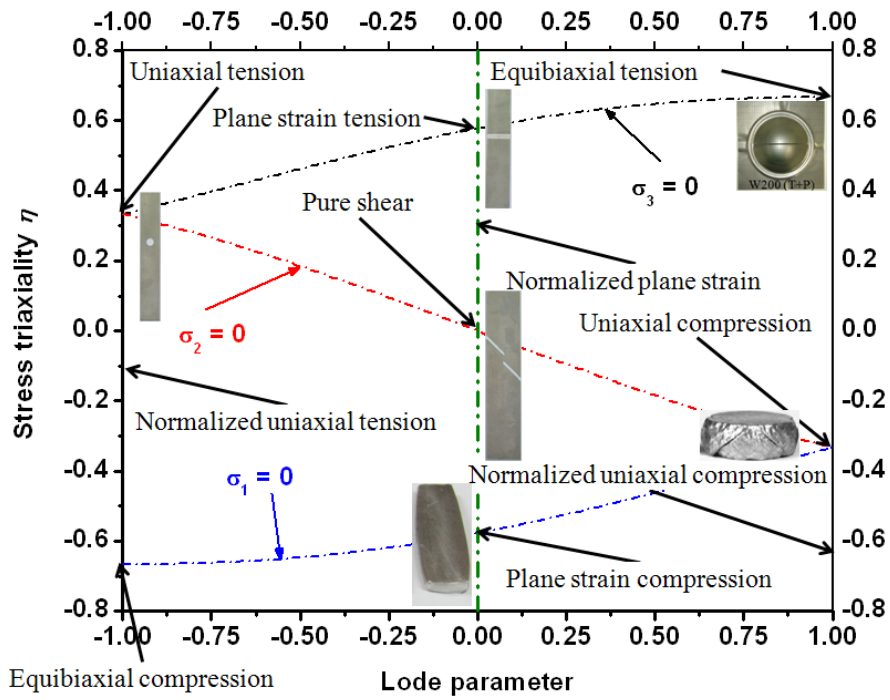

Figure 2: $\quad$ Stress states in the space of stress triaxiality and Lode parameter $(\eta, L)$.

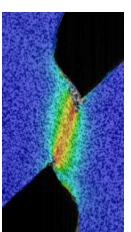

(a)

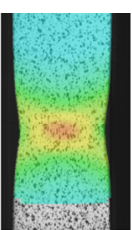

(b)

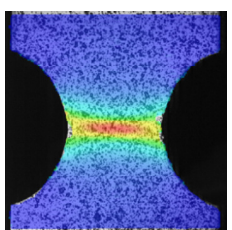

(c)

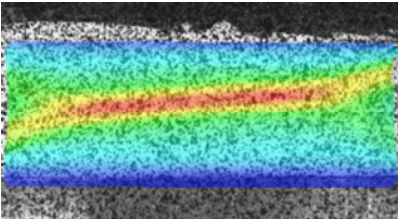

(d)

Figure 3: Calculated equivalent strain before the fracture (Ti6Al4V); (a) pure shear; (b) dog bone; (c) notched; (d) plane strain specimen.

software ARAMIS. Fig. 3 shows an example of the patterned specimen surface and calculated strain distribution before fracture.

The strain rate effect can be considered by changing the tensile speed of the test machine. The numerical simulation with each specimen is repeatedly carried out to find the tensile speed in order to obtain the equivalent strain rates as 0.001 , $0.1,1,10,100 \mathrm{~s}^{-1}$ at the fractured point. Table 3 shows the calculated tensile speed with respect to the strain rate condition.

Table 3: $\quad$ Tensile speed with respect to the strain rate $[\mathrm{mm} / \mathrm{s}]$.

\begin{tabular}{|c|c|c|c|c|c|}
\hline Strain rate & $\mathbf{0 . 0 0 1 s}^{-1}$ & $\mathbf{0 . 1 s}^{-1}$ & $\mathbf{1 s}^{-1}$ & $\mathbf{1 0 s}^{-1}$ & $\mathbf{1 0 0 s}^{-1}$ \\
\hline Pure shear & 0.004 & 0.4 & 4 & 40 & 400 \\
\hline Dog bone & 0.03 & 3 & 30 & 300 & 3000 \\
\hline Notched & 0.0038 & 0.38 & 3.8 & 38 & 380 \\
\hline Plane strain & 0.0025 & 0.25 & 2.5 & 25 & 250 \\
\hline
\end{tabular}




\subsection{Lou-Huh ductile fracture criterion}

The Lou-Huh ductile fracture criterion is proposed to describe ductile fracture behaviour of metals for nucleation, growth and shear coalescence of voids which has a form of:

$$
\int_{0}^{\bar{\varepsilon}_{f}}\left(\frac{2 \tau_{\max }}{\bar{\sigma}}\right)^{C_{1}}\left(\frac{\langle 1+3 \eta\rangle}{2}\right)^{C_{2}} d \bar{\varepsilon}=C_{3} \quad\langle x\rangle=\left\{\begin{array}{lll}
x & \text { when } & x \geq 0 \\
0 & \text { when } & x<0
\end{array}\right.
$$

The nucleation of voids is suggested as a function of equivalent plastic strain $\bar{\varepsilon}$, the growth of voids is calculated by the stress triaxiality as $1+3 \eta$ and the coalescence of voids is determined by the normalized maximum shear stress $\tau_{\max } / \bar{\sigma}$. The fracture strain predicted by the Lou-Huh ductile fracture criterion is negative dependent on the stress triaxiality. Eqn (1) also can be transformed into the space of as below:

$$
\int_{0}^{\bar{s}_{f}}\left(\frac{2}{\sqrt{L^{2}+3}}\right)^{C_{1}}\left(\frac{\langle 1+3 \eta\rangle}{2}\right)^{C_{2}} d \bar{\varepsilon}=C_{3} \quad\langle x\rangle=\left\{\begin{array}{rrr}
x & \text { when } & x \geq 0 \\
0 & \text { when } & x<0
\end{array}\right.
$$

The fracture strain, strain path and stress triaxiality of each specimen are calculated by the commercial software ARAMIS with the recorded pattern on the surface of specimen and the three coefficients are determined to reduce the difference between experimental data and the Lou-Huh criterion.

\section{Experiment result}

\subsection{Stress-strain curves and recommended dynamic hardening models}

Figs 4-6 show the stress-strain curves and variations of yield stress 4130 steel, OFHC copper and Ti6Al4V at the strain rates ranging from 0.001 to $100 \mathrm{~s}^{-1}$. The yield stress and the flow stress increase as the strain rate increases, but the amount and the tendency of the increment for the three materials are different from each other. When the strain rate increases, the yield stress of 4130 steel and OFHC copper also increase exponentially but that of Ti6Al4V increases linearly. The strain rate sensitivity of 4130 steel is quite different from that of OFHC copper. Due to these characteristics, the stress-strain curves can be accurately fitted by the Lim-Huh model (4130 steel), the Preston-Tonks-Wallace model (OFHC copper) and the modified Khan-Huang model (Ti6Al4V) as below:

The Lim-Huh model [5]

$$
\sigma=\sigma_{r} \frac{1+q(\varepsilon) \dot{\varepsilon}^{m(e)}}{1+q(\varepsilon) \dot{\varepsilon}_{r}^{m(e)}} \quad \text { where } \quad q=C^{-\frac{1}{p}}, m=\frac{1}{p}
$$


The Preston-Tonks-Wallace (PTW) model

$$
\begin{aligned}
& \hat{\tau}=\hat{\tau}_{s}+\frac{1}{p}\left(s_{0}-\hat{\tau}_{y}\right) \ln \left[1-\left[1-\exp \left(-p \frac{\hat{\tau}_{s}-\hat{\tau}_{y}}{s_{0}-\hat{\tau}_{y}}\right)\right] \times \exp \left\{-\frac{p \theta \psi}{\left(s_{0}-\hat{\tau}_{y}\right)\left[\exp \left(-p \frac{\hat{\tau}_{s}-\hat{\tau}_{y}}{s_{0}-\hat{\tau}_{y}}\right)-1\right]}\right\}\right] \\
& \text { where }\left\{\begin{array}{l}
\hat{\tau}_{s}=s_{0}-\left(s_{0}-s_{\infty}\right) \operatorname{erf}[\kappa \hat{T} \ln (\gamma \dot{\xi} / \dot{\psi})] \\
\hat{\tau}_{y}=y_{0}-\left(y_{0}-y_{\infty}\right) \operatorname{erf}[\kappa \hat{T} \ln (\gamma \dot{\xi} / \dot{\psi})]
\end{array}\right.
\end{aligned}
$$

The modified Khan-Huang model [6]

$$
\sigma=\left[a+B\left(1-\frac{\ln \dot{\varepsilon}}{\ln D_{0}^{p}}\right)^{n_{1}} \varepsilon^{n_{0}}\right]\left[1+C\left(\ln \frac{\dot{\varepsilon}}{\dot{\varepsilon}_{0}}\right)^{p}\right]\left[1-\left(\frac{T-T_{r}}{T-T_{m}}\right)^{m}\right]
$$

where $D_{0}^{p}=10^{9} / s$

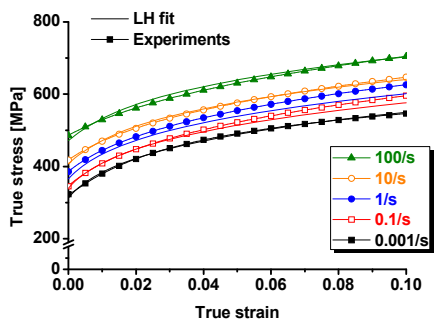

(a)

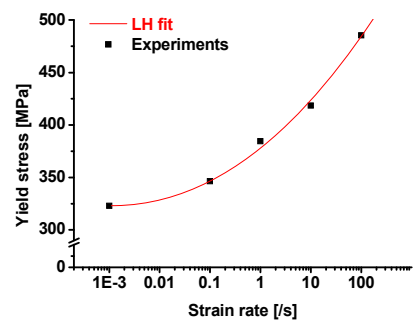

(b)

Figure 4: (a) the stress-strain curve and (b) the variation of the yield stress of 4130 steel with respect to the strain rate. The results are fitted by Lim-Huh model.

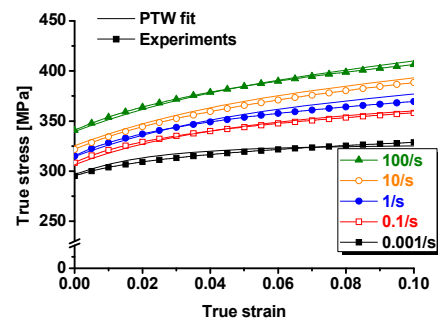

(a)

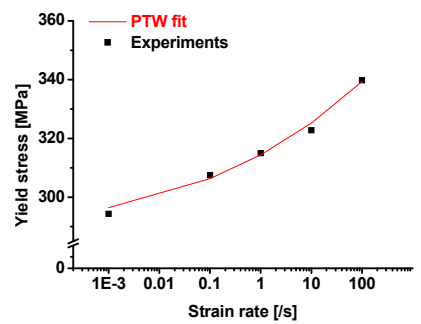

(b)

Figure 5: (a) the stress-strain curve and (b) the variation of the yield stress of OFHC copper with respect to the strain rate. The results are fitted by PTW model. 


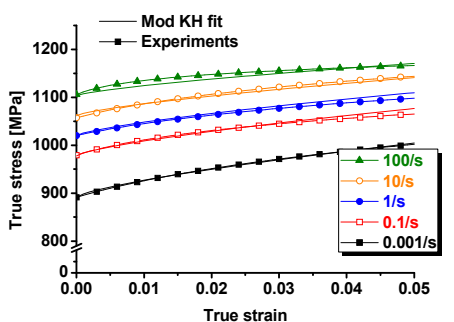

(a)

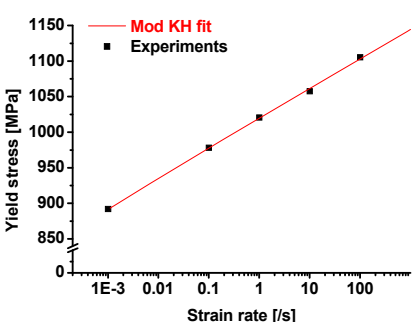

(b)

Figure 6: (a) the stress-strain curve and (b) the variation of the yield stress of Ti6Al4V with respect to the strain rate. The results are fitted by modified Khan-Huang model.

\subsection{Fracture strain and fracture loci with respect to the strain rate}

No ductile fracture model considering both the strain rate and the loading path has been reported yet. However, the fracture strain can be calibrated by the LouHuh model without considering the strain rate and then the dependence on the strain rate can be separately considered to construct a new model which incorporates both the fracture strain and the strain rate. Fig. 7 indicates where the fracture strains are measured at the four points of different stress triaxiality conditions and fitted by the Lou-Huh model. And then the result can be transformed into the forming limit diagram. Figs 8-10 show the fracture loci with respect to the strain rate in $\left(\bar{\varepsilon}_{f}, \eta\right)$ and $\left(\varepsilon_{1}, \varepsilon_{2}\right)$ space. As the strain rate increases from 0.001 to $100 \mathrm{~s}^{-1}$, the fracture strain under the uniaxial tension condition has the minimum strain at the strain rate of $1 \mathrm{~s}^{-1}$ and the maximum strain at the $100 \mathrm{~s}^{-1}$. This overlap is observed in all materials. However, the fracture strain under the pure shear condition shows quite different tendency with respect to the strain rate.

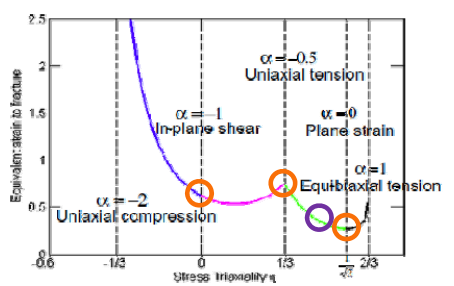

(a)

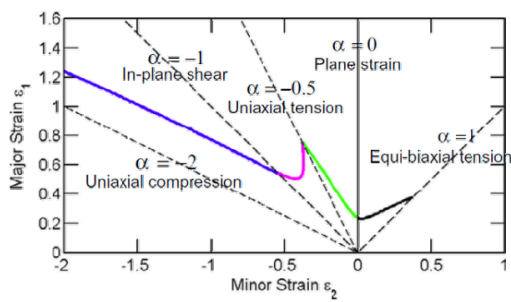

(b)

Figure 7: (a) fracture locus under plane stress condition (b) transformed fracture forming limit diagram. 


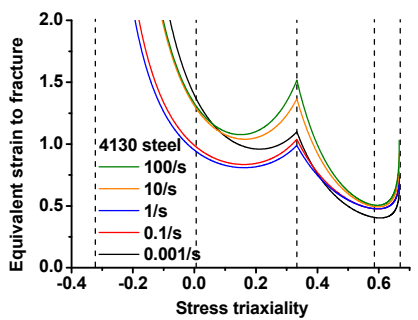

(a)

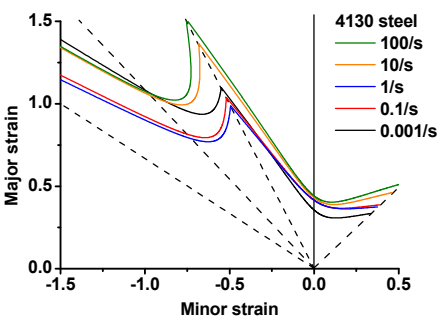

(b)

Figure 8: (a) the fracture loci, (b) fracture forming limit diagram of 4130 steel according to the strain rate.

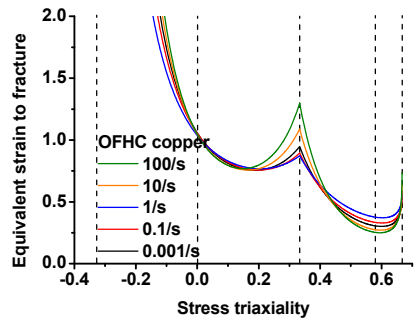

(a)

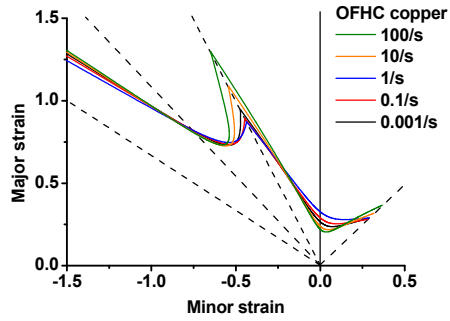

(b)

Figure 9: (a) the fracture loci, (b) fracture forming limit diagram of OFHC copper according to the strain rate.

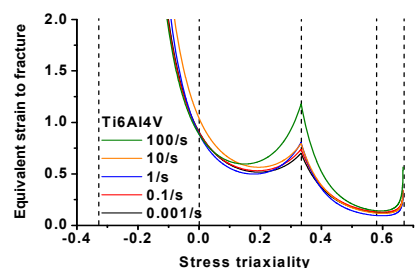

(a)

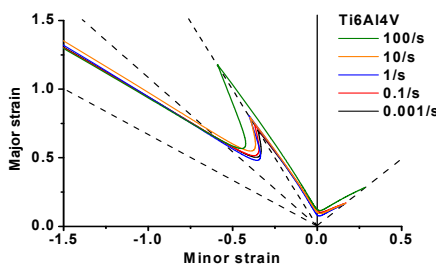

(b)

Figure 10: (a) the fracture loci, (b) fracture forming limit diagram of Ti6Al4V according to the strain rate.

\section{Conclusion}

The dynamic properties of the three metallic materials are investigated with respect to the strain rate. The three metallic materials show different flow stress tendencies. The Lim-Huh model, the PTW model and the modified Khan-Huang model are most applicable for 4130 steel, OFHC copper and Ti6Al4V respectively. The fracture strains are measured on the surface of a specimen with 
respect to the strain rate and loading path. The fracture strain under the uniaxial tension condition has the minimum value at the strain rates ranging from 0.1 to $1 \mathrm{~s}^{-1}$ and the maximum value at $100 \mathrm{~s}^{-1}$. However the fracture strain under the pure shear condition shows quite different tendency with respect to the strain.

\section{References}

[1] D. L. Preston, D. L. Tonks, D. C. Wallace, Model of plastic deformation for extreme loading conditions, Journal of Applied Physics, 93, pp. 211-220, 2003.

[2] Y. L. Bai and T. Wierzbicki, Forming severity concept for predicting sheet necking under complex loading histories, Int. J. Mech. Sci., 50, pp. 10121022, 2008.

[3] Y. B. Bao and T. Wierzbicki, A comparative study on various ductile crack formation criteria, J. Eng. Mater. Tech. ASME, 126, pp. 314-324, 2004.

[4] Y. Lou, H. Huh, S. Lim, K. Pack, New ductile fracture criterion for prediction of fracture forming limit diagrams of sheet metals, International journal of solid and structure, 49, pp. 3605-3615, 2012.

[5] J. H. Lim, Study on dynamic tensile tests of auto-body steel sheets at the intermediate strain rate for material constitutive equations, Ph.D Dissertation, KAIST, 2005.

[6] H. J. Lee, J. H. Song, H. Huh, Dynamic tensile tests of auto-body steel sheets with the variation of temperature, Solid state phenomena, 114-117, pp. 259-262, 2006. 